\title{
Research Article \\ On the Variational Eigenvalues Which Are Not of Ljusternik-Schnirelmann Type
}

\author{
Pavel Drábek \\ Department of Mathematics and N.T.I.S., University of West Bohemia, Univerzitní 22, \\ 30614 Plzen̆, Czech Republic \\ Correspondence should be addressed to Pavel Drábek, pdrabek@kma.zcu.cz
}

Received 29 November 2011; Revised 12 January 2012; Accepted 12 January 2012

Academic Editor: Donal O’Regan

Copyright (C) 2012 Pavel Drábek. This is an open access article distributed under the Creative Commons Attribution License, which permits unrestricted use, distribution, and reproduction in any medium, provided the original work is properly cited.

\begin{abstract}
We discuss nonlinear homogeneous eigenvalue problems and the variational characterization of their eigenvalues. We focus on the Ljusternik-Schnirelmann method, present one possible alternative to this method and compare it with the Courant-Fischer minimax principle in the linear case. At the end we present a special nonlinear eigenvalue problem possessing an eigenvalue which allows the variational characterization but is not of Ljusternik-Schnirelmann type.
\end{abstract}

The question of characterization of the set of all eigenvalues of nonlinear homogeneous eigenvalue problem is a very important problem of the calculus of variations that has been open since the 1970s, see, for example, [1]. While the well-known Courant-Fischer minimax principle provides complete characterization of the set of eigenvalues of the linear eigenvalue problem, its nonlinear counterparts, like the Ljusternik-Schnirelmann method, do not exhaust the entire set of eigenvalues in general. The purpose of this paper is to discuss this issue in the context of general homogeneous nonlinear eigenvalue problem (1). In particular, we want to explain that "variational characterization" is not a synonym for "Ljusternik-Schnirelmann characterization." Indeed, this obfuscation is quite common in the literature (cf. [2]) and might lead to many misunderstandings.

We present a variational argument in order to find an eigenvalue that is not characterized by the Ljusternik-Schnirelmann formula. For this purpose we present the periodic problem for the one-dimensional $p$-Laplacian from [3] as a concrete example and heuristically enlighten technically involved estimates. We show the local character of our variational characterization contrary to the global character of the Ljusternik-Schnirelmann method. This illustrates the striking difference between the linear and nonlinear problems. Note that also this "local procedure" does not yield all the eigenvalues of the problem in question. 
The novelty of this paper is thus to point out some connections might be hidden in more technically complicated papers and to remind some challenging problems of the calculus of variations. We also rigorously show that the second eigenvalue of LjusternikSchnirelmann type for (1) can be found as a minimax over a special class of curves rather than the family of sets of genus greater than or equal to 2 . This fact makes the construction of the second eigenvalue more geometrically transparent and might be useful in numerical approach to find its concrete value.

Let $J, S: X \rightarrow X^{*}$ be odd and $(p-1)$-homogeneous operator from a Banach space $X$ into its dual, $p>1$. A real number $\lambda$ and an element $u \in X, u \neq 0$, for which

$$
J(u)-\lambda S(u)=0
$$

are called an eigenvalue and an eigenvector of the eigenvalue problem (1). Set

$$
F(u):=\langle J(u), u\rangle, \quad G(u):=\langle S(u), u\rangle,
$$

where $\langle\cdot, \cdot\rangle$ stands for the duality pairing between $X^{*}$ and $X$. Assume that $J$ and $S$ are such that $F$ and $G$ are $C^{1}$-functionals from $X$ into $\mathbb{R}$,

$$
\begin{gathered}
\left\langle F^{\prime}(u), v\right\rangle=\langle J(u), v\rangle,\left\langle G^{\prime}(u), v\right\rangle=\langle S(u), v\rangle, \\
\mathcal{S}:=\{u \in X: G(u)=1\}
\end{gathered}
$$

is a $C^{1}$-manifold without boundary that does not contain the zero element $0 \in X$. The Lagrange multiplier method implies that $\lambda_{0}$ is an eigenvalue of (1) and $u_{0}$ is the corresponding eigenvector if and only if $u_{0}$ is a critical point of $\left.F\right|_{S}$ and $F\left(u_{0}\right)=\lambda_{0}$ (i.e., $\lambda_{0}$ is the corresponding critical level). There are several methods to find critical points of $F$ on $\mathcal{S}$. However, it seems that with the exception of some special situations (e.g., $J$ and $S$ are linear operators) none of the "nonlinear" methods describes the entire set of critical levels and critical points of $\left.F\right|_{S}$. One of the most frequently used methods is based on the notion of the Krasnoselskii genus.

Set $\mathcal{A}:=\{A \subset X: A$ is closed and symmetric $\}$. The Krasnoselskii genus of a set $A$, $\gamma(A)$, is defined as follows:

$$
\gamma(A):=\left\{\begin{array}{l}
\inf \left\{m \in \mathbb{N}: \exists h \in C^{0}\left(A, \mathbb{R}^{m} \backslash\{0\}\right), h \text { is odd }\right\} \\
\infty \text { if }\left\{m \in \mathbb{N}: \exists h \in C^{0}\left(A, \mathbb{R}^{m} \backslash\{0\}\right), h \text { is odd }\right\}=\emptyset
\end{array}\right.
$$

For any $k \in \mathbb{N}$ consider a family of sets defined by

$$
\mathcal{F}_{k}:=\{A \in \mathcal{A}: A \subset \mathcal{S}, \gamma(A) \geq k\}
$$

Assume that $\gamma(\mathcal{S})=\infty$. Then, $\mathscr{\mp}_{k} \neq \emptyset$ for any $k \in \mathbb{N}$ and it is invariant under any continuous and odd map from $\mathcal{S}$ into itself.

Let us assume that $F$ satisfies the Palais-Smale condition on $\mathcal{S}$. The LjusternikSchnirelmann method yields a sequence $\left\{\lambda_{k}\right\}_{k=1}^{\infty} \subset \mathbb{R}$ given by

$$
\lambda_{k}:=\inf _{A \in \mathscr{F}_{k}} \sup _{u \in A} F(u)
$$


Every $\lambda_{k}$ is a critical level of $F$ subject to $\mathcal{S}$, and it is achieved at some $u_{k} \in \mathcal{S}$, that is,

$$
\left(\left.F\right|_{S}\right)^{\prime}\left(u_{k}\right)=0
$$

(cf. [4, 5]). It follows that $\lambda_{k}$ is an eigenvalue of (1) and $u_{k}$ is the corresponding eigenvector. In general, the sequence $\left\{\lambda_{k}\right\}_{k=1}^{\infty}$ given by (6) does not exhaust the set of all critical levels of $\left.F\right|_{S}$, and thus it might not be the set of all eigenvalues of (1). An eigenvalue of (1) that allows the characterization (6) is called an eigenvalue of Ljusternik-Schnirelmann type.

The model example of the abstract setting presented above is the eigenvalue problem for the Dirichlet $p$-Laplacian. Indeed, set $X=W_{0}^{1, p}(\Omega), p>1$, and

$$
F(u)=\int_{\Omega}|\nabla u|^{p} \mathrm{~d} x, \quad G(u)=\int_{\Omega}|u|^{p} \mathrm{~d} x,
$$

where $\Omega \subset \mathbb{R}^{N}$ is a bounded domain. Then,

$$
\mathcal{S}=\left\{u \in X:\|u\|_{L^{p}(\Omega)}=1\right\}
$$

and a critical point $u_{0} \in \mathcal{S}, F\left(u_{0}\right)=\lambda_{0}$ satisfies

$$
\left\langle F^{\prime}\left(u_{0}\right), v\right\rangle=\lambda_{0}\left\langle G^{\prime}\left(u_{0}\right), v\right\rangle
$$

for any $v \in X$. But this is a weak formulation of the problem

$$
\begin{gathered}
-\Delta_{p} u_{0}-\lambda_{0}\left|u_{0}\right|^{p-2} u_{0}=0 \quad \text { in } \Omega, \\
u_{0}=0 \quad \text { on } \partial \Omega,
\end{gathered}
$$

and, therefore, $\lambda_{0}$ is an eigenvalue and $u_{0}$ is the corresponding eigenvector (eigenfunction) of the Dirichlet $p$-Laplacian (11).

If $p \neq 2$ and $\Omega \subset \mathbb{R}^{N}, N \geq 2$, it is a long-standing open problem whether or not the sequence $\left\{\lambda_{k}\right\}_{k=1}^{\infty}$ given by (6) exhausts the entire set of eigenvalues of (11). On the other hand, if $\Omega=(0,1), p>1$, or $\Omega \subset \mathbb{R}^{N}, N \geq 1$, and $p=2$ (i.e., the problem (11) is linear), the sequence $\left\{\lambda_{n}\right\}_{n=1}^{\infty}$ given by (6) is the set of all eigenvalues of (11). To prove this fact in the former case the ODE techniques (uniqueness of the solution of an associated initial value problem) are employed while in the latter case the linearity of the problem plays the key role.

There are several other variational characterizations similar to (6). Namely, the family of sets $\mathcal{F}_{k}$ can be replaced by an other family $\tilde{\boldsymbol{F}}_{k}$, which is invariant with respect to any continuous odd semiflow $\Phi: \mathcal{S} \times[0, \infty) \rightarrow \mathcal{S}$ such that $\Phi(\cdot, 0)=\operatorname{id}_{\mathcal{S}}, \Phi(\cdot, t): \mathcal{S} \rightarrow \mathcal{S}$ is a homeomorphism for any fixed $t \geq 0$ and $F(\Phi(u, \cdot))$ is nonincreasing for any fixed $u \in \mathcal{S}$. 
Then,

$$
\tilde{\jmath}_{k}:=\inf _{A \in \tilde{\mathscr{Y}}_{k}} \sup _{u \in A} F(u), \quad k \in \mathbb{N}
$$

is a critical level of $\left.F\right|_{S}$.

We give a concrete example of $\tilde{\mathscr{F}}_{k}$ below (cf. [6]). Let $S^{k-1}$ be the unit sphere in $\mathbb{R}^{k}$.

Set

$$
\tilde{\Psi}_{k}:=\left\{A \subset \mathcal{S}: \exists h: S^{k-1} \longrightarrow \mathcal{S}, A=h\left(S^{k-1}\right), h \text { is odd and continuous }\right\}
$$

It is clear that $\tilde{\mathscr{f}}_{k} \subsetneq \mathcal{F}_{k}$, which implies

$$
\lambda_{k} \leq \tilde{\lambda}_{k}, \quad k \in \mathbb{N}
$$

It is also obvious that $\lambda_{1}=\tilde{\lambda}_{1}$. We claim that $\lambda_{2}=\tilde{\lambda}_{2}$ as well. Indeed, for an $A \in \mathcal{F}_{2}$ there exists (arbitrarily small) symmetric neighborhood $\mathcal{N}(A) \subset \mathcal{S}$ such that $\gamma(\overline{\mathcal{N}(A)}) \geq 2$ (see, [7, Proposition 2.3]). Without loss of generality we may assume that $\overline{\mathcal{N}(A)}$ is a pathwise connected set in $\mathcal{S}$ (otherwise $\gamma(\overline{\mathcal{N}(A)})=1$, a contradiction). Choosing $u \in \overline{\mathcal{N}(A)}$, we have $-u \in \overline{\mathcal{N}(A)}$ by symmetry, and there is a continuous path $\varphi \in \overline{\mathcal{N}(A)}$ from $u$ to $-u$. By symmetry of $\overline{\mathcal{N ( A )}}$ we also have $-\varphi \subset \overline{\mathcal{N ( A )}}$. But $\varphi \cup(-\varphi)=h\left(S^{1}\right)$ for some continuous and odd map $h$ of $S^{1}$ into $\mathcal{S}$. Since the neighborhood $\mathcal{N}(A)$ can be chosen small enough, the value of

$$
\max _{u \in \varphi \cup(-\varphi)} F(u)-\sup _{u \in A} F(u)
$$

can be made smaller than any positive number. This implies $\tilde{\lambda}_{2} \leq \lambda_{2}$, which proves the claim.

The author is not aware of any assumptions laid on $J$ and $S$ that would guarantee that also $\lambda_{k}=\tilde{\lambda}_{k}$ for $k \geq 3$ (cf. [6]). principle:

It is instructive to compare formula (12) with the classical Courant-Fisher minimax

$$
c_{k}:=\inf _{\substack{X^{\prime} \subset X \\ \operatorname{dim} X^{\prime}=k}} \max _{u \in \mathcal{S} \cap X^{\prime}} F(u)
$$

under the assumptions that $J, S$ are linear operators, $X$ is a Hilbert space and $(J u, u)_{X}$ defines an equivalent inner product on $X$ (cf. problem (11) with $p=2$ ).

Let $X^{\prime} \subset X, \operatorname{dim} X^{\prime}=k$. Then, $A=\mathcal{S} \cap X^{\prime}$ is homeomorphic to $S^{k-1}$, that is, $A \in \tilde{\mathscr{F}}_{k}$ and hence $\tilde{\lambda}_{k} \leq c_{k}$. In order to prove the opposite inequality we need the following assertion.

Lemma 1 (cf. [5]). Any compact and symmetric subset $A$ of a Hilbert space, such that $\gamma(A)=k$ contains at least $k$ mutually orthogonal vectors $u_{1}, \ldots, u_{k}$. 
Since $\gamma(A)=k$ for $A \in \tilde{F}_{k}$, there exist $u_{1}, \ldots, u_{k} \in A$ such that $\left(J u_{i}, u_{j}\right)_{X}=0$ for $i \neq j$. Set $X^{\prime}=\operatorname{Lin}\left\{u_{1}, \ldots, u_{k}\right\}$. It follows from the linearity of $J$ that $\max _{\mathcal{S} \cap X^{\prime}} F(u) \leq \max _{A} F(u)$, which implies $c_{k} \leq \tilde{\mathcal{\lambda}}_{k}$. Together with the opposite inequality proved above we thus get

$$
\tilde{\lambda}_{k}=c_{k}
$$

Similarly, one proves that also $\lambda_{k}=c_{k}$.

In particular, it follows from our discussion that the second Ljusternik-Schnirelmann eigenvalue of (1) can be characterized as follows:

$$
\lambda_{2}=\inf _{r \in \mathcal{C}_{\text {sym }}} \max _{u \in \gamma} F(u)
$$

where $\mathcal{C}_{\text {sym }}$ is the family of all continuous symmetric and closed curves in $\mathcal{S}$.

The idea of construction of an eigenvalue that is not of Ljusternik-Schnirelmann type comes from the following heuristics.

Set $\Phi(\gamma):=\max _{u \in \gamma} F(u)$. Then, $\lambda_{2}=\inf _{\gamma \in \mathcal{C}_{\text {sym }}} \Phi(\gamma)$, that is, $\lambda_{2}$ is a "global minimum" of $\Phi$ over the set $\mathcal{C}_{\text {sym }}$ (cf. [5]). The idea is to find a "local minimum" of $\Phi$ over $\mathcal{C}_{\text {sym }}$, say $\mu_{2}$, which satisfies $\lambda_{2}<\mu_{2}<\lambda_{3}$, that is, $\mu_{2} \notin\left\{\lambda_{k}\right\}_{k=1}^{\infty}$. For this purpose we find a subset of curves $\mathcal{C} \subset \mathcal{C}_{\text {sym }}$, which is invariant with respect to any continuous odd semiflow and which satisfies

$$
\lambda_{2}=\inf _{\gamma \in \mathcal{C}_{\text {sym }}} \max _{u \in \gamma} F(u)<\inf _{\gamma \in \mathcal{C}} \max _{u \in \gamma} F(u)=\mu_{2}<\lambda_{3}
$$

The invariance of $\mathcal{C}$ then guarantees that $\mu_{2}$ is a critical point of $\left.F\right|_{S}$.

Below we present a concrete example. The technical details and precise estimates can be found in [3]. We set $X=W_{\text {per }}^{1, p}\left(-\pi_{p}, \pi_{p}\right)$, the Sobolev space of all $\left(2 \pi_{p}\right)$-periodic functions $f: \mathbb{R} \rightarrow \mathbb{R}$ such that the restriction of $f$ to the interval $\left[-\pi_{p}, \pi_{p}\right]$ belongs to $W^{1, p}\left(-\pi_{p}, \pi_{p}\right)$ and $f\left(-\pi_{p}\right)=f\left(\pi_{p}\right)$. This space is endowed with the norm

$$
\|f\|:=\left(\left\|f^{\prime}\right\|_{L^{p}\left(-\pi_{p}, \pi_{p}\right)}^{p}+\|f\|_{L^{p}\left(-\pi_{p}, \pi_{p}\right)}^{p}\right)^{1 / p}<\infty
$$

Here,

$$
\pi_{p}:=2 \int_{0}^{1} \frac{\mathrm{d} s}{\left(1-s^{p}\right)^{1 / p}}=\frac{2 \pi}{p \sin (\pi / p)}
$$

We also set

$$
\mathcal{S}:=\left\{f \in X:\|f\|_{L^{p}\left(-\pi_{p}, \pi_{p}\right)}=1\right\}
$$

Let $\varepsilon>0$ be a fixed number, and let $q: \mathbb{R} \rightarrow \mathbb{R}$ be a given continuous $\left(2 \pi_{p}\right)$-periodic function of $x \in \mathbb{R}$. 
We define $J_{\varepsilon}, S: X \rightarrow X^{*}$ by

$$
\begin{aligned}
\left\langle J_{\mathcal{E}}(u), v\right\rangle & :=\int_{-\pi_{p}}^{\pi_{p}}\left|u^{\prime}\right|^{p-2} u^{\prime} v^{\prime} \mathrm{d} x+\varepsilon \int_{-\pi_{p}}^{\pi_{p}} q|u|^{p-2} u v \mathrm{~d} x, \\
\langle S(u), v\rangle & :=\int_{-\pi_{p}}^{\pi_{p}}|u|^{p-2} u v \mathrm{~d} x
\end{aligned}
$$

for $u, v \in X$. We also set

$$
F_{\varepsilon}(u):=\int_{-\pi_{p}}^{\pi_{p}}\left|u^{\prime}\right|^{p} \mathrm{~d} x+\varepsilon \int_{-\pi_{p}}^{\pi_{p}} q|u|^{p} \mathrm{~d} x
$$

The operator equation

$$
J_{\mathcal{\varepsilon}}(u)-\lambda S(u)=0
$$

corresponds to the weak formulation of the following eigenvalue problem:

$$
\begin{gathered}
-\left(\left|u^{\prime}\right|^{p-2} u^{\prime}\right)^{\prime}+\varepsilon q(x)|u|^{p-2} u=\lambda|u|^{p-2} u \text { in } \mathbb{R}, \\
u \text { is }\left(2 \pi_{p}\right) \text {-periodic in } \mathbb{R} .
\end{gathered}
$$

For $\varepsilon=0$ the set of all eigenvalues of (26) is given by the sequence

$$
\lambda_{0}=0, \quad \lambda_{2 n-1}=\lambda_{2 n}=(p-1) n^{p}, \quad n=1,2, \ldots,
$$

and it is proved in [2] that each eigenvalue $\lambda_{k}(k=0,1,2, \ldots)$ has a Ljusternik-Schnirelmann variational characterization:

$$
\lambda_{k}=\inf _{A \in \mathscr{T}_{k+1}} \max _{u \in A} \int_{-\pi_{p}}^{\pi_{p}}\left|u^{\prime}(x)\right|^{p} \mathrm{~d} x
$$

The eigenvalue $\lambda_{0}$ is simple, and the corresponding eigenfunction is a constant (and thus does not change the sign in $\left[-\pi_{p}, \pi_{p}\right]$ ). For any $n=1,2, \ldots$, we have that $\lambda_{2 n-1}=\lambda_{2 n}$ is not a simple eigenvalue. Given any fixed $t \in \mathbb{R}$, every function $\sin _{p}(n x+t)$ of $x \in \mathbb{R}$ is an eigenfunction associated with $\lambda_{2 n-1}=\lambda_{2 n}$. Here, $\sin _{p} x$ for $x \in\left[0, \pi_{p} / 2\right]$ is defined implicitly by the formula

$$
x=\int_{0}^{\sin _{p} x}\left(1-s^{p}\right)^{-1 / p} \mathrm{~d} s
$$

and then extended to an odd (about 0$),\left(2 \pi_{p}\right)$-periodic function on $\mathbb{R}$.

Remark 2. The reader should notice that only in the linear case $p=2$ do the corresponding eigenfunctions associated with $\lambda_{2 n-1}=\lambda_{2 n}$ form a linear space of dimension two. In contrast, if 
$p \neq 2$, then the multiplicity should be understood in the sense of the Ljusternik-Schnirelmann variational characterization (28). In fact, in this case the set of all eigenfunctions associated with each eigenvalue $\lambda_{2 n-1}=\lambda_{2 n}$ is a two-dimensional manifold, the linear hull of which is infinitedimensional (cf. [2]). If these eigenfunctions are normalized by a scalar multiplication factor in order to belong to $\mathcal{S}$, they form a one-dimensional submanifold in $L^{p}\left(-\pi_{p}, \pi_{p}\right)$, which is diffeomorphic to a circle, the linear hull of which is again infinite-dimensional.

For $\varepsilon>0, q \neq 0$ the problem (26) possesses a sequence of eigenvalues tending to infinity and given by

$$
\lambda_{k}^{\varepsilon}=\inf _{A \in \Psi_{k+1}} \max _{u \in A} F_{\varepsilon}(u)
$$

for $k=0,1,2, \ldots$. The first eigenvalue is simple, and the corresponding eigenfunction does not change sign. For each $n=1,2,3, \ldots$ we have

$$
\lambda_{2 n-1}^{\varepsilon} \leq \lambda_{2 n}^{\varepsilon}<\lambda_{2 n+1}^{\varepsilon} \leq \lambda_{2 n+2}^{\varepsilon}
$$

where equalities may possibly occur. Eigenfunctions associated with $\lambda_{2 n-1}^{\varepsilon}$ and $\lambda_{2 n}^{\varepsilon}$ have precisely $2 n$ zeros in $\left(-\pi_{p}, \pi_{p}\right]$, and, for each $k=0,1,2, \ldots$, we have

$$
\lim _{\varepsilon \rightarrow 0+} \lambda_{k}^{\varepsilon}=\lambda_{k}
$$

(see [2]). It is also proved in [2] that for any integers $m, n \geq 1$ and for any $\varepsilon>0$, small enough, there exists a special $\left(2 \pi_{p}\right)$-periodic function $q \in C^{1}(\mathbb{R})$ such that $\lambda_{2 n-1}^{\varepsilon}<\lambda_{2 n}^{\varepsilon}$ and the open interval $\left(\lambda_{2 n-1}^{\varepsilon}, \lambda_{2 n}^{\varepsilon}\right)$ contains at least $m$ eigenvalues of (26). In particular, these eigenvalues do not allow the variational characterization (30). In the paper [3] we have shown that in contrast with the eigenvalues of Ljusternik-Schnirelmann type that are "globally variational," some of the above mentioned "new" eigenvalues from $\left(\lambda_{2 n-1}, \lambda_{2 n}\right)$ have a variational characterization that has a "local character," meaning that the minimum part (inf) of the minimax formula (30) is taken only locally in the sense mentioned above. In order to illustrate our method we focus on $n=1$, that is, we find "local variational" eigenvalue of (26) in the interval $\left(\lambda_{1}^{\varepsilon}, \lambda_{2}^{\varepsilon}\right)$, provided $\varepsilon$ is small enough.

The first critical level of Ljusternik-Schnirelmann type (L.S. type for short) for $F_{0}$ on $\mathcal{S}, 0=\lambda_{0}=\min _{u \in \mathcal{S}} F_{0}(u)$ is attained at $\pm \varphi_{0}\left(\varphi_{0} \equiv\right.$ constant $\left.>0\right)$. The second critical level of L.S. type, $\lambda_{1}=p-1$, is defined by the minimax formula

$$
\lambda_{1}=\min _{\gamma \in \mathcal{C}_{1}} \max _{\tau \in[-1,1]} F_{0}(\gamma(\tau))
$$

where $\mathcal{C}_{1}$ is the set of all continuous curves in $\mathcal{S}$ that connect the "south pole"- $\varphi_{0}$ with the "north pole" $\varphi_{0}$. The level $\lambda_{1}$ is attained at the point $u=\gamma_{t}(0)$ on the curve $\gamma_{t} \in \mathcal{C}_{1}(t \in \mathbb{R})$ defined by

$$
\gamma_{t}(\tau):=\left\|h_{t}(\tau)\right\|_{L_{\mathrm{per}}^{p}} h_{t}(\tau), \quad \tau \in[-1,1]
$$


where

$$
h_{t}(\tau)=\tau \varphi_{0}+\left(1-\tau^{2}\right) e(t)(x), \quad x \in \mathbb{R}
$$

and $e(t)(x):=\left(2 \pi_{p} / p\right)^{-1 / p} \sin _{p}(x+t)$ is a normalized eigenfunction associated with $\lambda_{1}=\lambda_{2}$ (see [3]). Notice that the curves $\gamma_{t}$ correspond to the "meridians" on $\mathcal{S}$ with the points of "longitude" $t$ and the "latitude" $\tau$.

For construction of a special function $q=q(x)$ the following observation plays the key role: the function $\sin _{p}: x \rightarrow \sin _{p} x$, from $\mathbb{R}$ to $\mathbb{R}$, is real analytic in the open set $\mathbb{R} \backslash\left\{(1 / 2) k \pi_{p}\right.$ : $k \in \mathbb{Z}\}$ and, furthermore, it is not $C^{3}$ at the point $k \pi_{p}(k \in \mathbb{Z})$ if $1<p<2$ and it is not $C^{3}$ at the point $(k+1 / 2) \pi_{p}$ if $(k \in \mathbb{Z}) 2<p<\infty$. Due to these facts a function $q$ can be found that controls the "splitting process" that involves the eigenvalues $\lambda_{1}^{\varepsilon}$ and $\lambda_{2}^{\varepsilon}$. In particular, $q$ is constructed in such a way that the function of the independent variable $t$,

$$
F(t ; q)=\int_{-\pi_{p}}^{\pi_{p}} q(x)\left|\sin _{p}(x+t)\right|^{p} \mathrm{~d} x
$$

has a global minimizer $t_{\min } \in\left[0, \pi_{p}\right]$ and a local minimizer $\bar{t}_{\min } \in\left(0, \pi_{p}\right)$ such that

$$
F\left(t_{\min } ; q\right)<F\left(\bar{t}_{\min } ; q\right)
$$

This property together with fine estimates for $p>2$ (see [3]) allows us to prove the local hyperbolic geometry of the functional $F_{\varepsilon}$ on $\mathcal{S}$. Namely, thanks to (37), we can "localize" the set of curves $\mathcal{C}_{2} \subsetneq \mathcal{C}_{1}$ that pass through a suitable open neighborhood of the function $e\left(\bar{t}_{\text {min }}\right)$ in the topology of $L^{p}\left(-\pi_{p}, \pi_{p}\right)$. The "inf" in the variational characterization of $\lambda_{1}^{\varepsilon}$ is then approached "away from" curves that belong to $\mathcal{C}_{2}$. The family of curves $\mathcal{C}_{2}$ is then proved to be invariant with respect to continuous odd semiflow on $\mathcal{S}$ and

$$
\lambda_{1}^{\varepsilon}=\inf _{\gamma \in \mathcal{C}_{1}} \max _{\tau \in[-1,1]} F_{\varepsilon}(\gamma(\tau))<\inf _{\gamma \in \mathcal{C}_{2}} \max _{\tau \in[-1,1]} F_{\varepsilon}(\gamma(\tau))<\lambda_{2}^{\varepsilon}
$$

holds for $\varepsilon>0$ small enough. Then,

$$
\lambda_{1}^{\varepsilon}<\tilde{\lambda}_{1}^{\varepsilon}=\inf _{\gamma \in \mathcal{C}_{2}} \max _{\tau \in[-1,1]} F_{\varepsilon}(\gamma(\tau))<\lambda_{2}^{\varepsilon}
$$

is the desired variational eigenvalue that is not of the L.S. type.

This construction is not possible in the linear case, $p=2$. Besides the fact that all eigenvalues of the linear problem are of L.S. type, this follows from the analyticity of the function $\sin x$. For this reason the functional $F$ with desired properties does not exist in the case $p=2$, which illustrates the striking difference between the linear and nonlinear eigenvalue problem. 


\section{Acknowledgment}

This work was supported by the European Regional Development Fund (ERDF), project "NTIS-New Technologies for Information Society," European Center of Excellence, CZ.1.05/1.1.00/02.0090 and project KONTAKT ME09109.

\section{References}

[1] S. Fučík, J. Nečas, J. Souček, and V. Souček, Spectral Analysis of Nonlinear Operators, vol. 346 of Lecture Notes in Mathematics, Springer, Berlin, Germany, 1973.

[2] P. Binding and B. Rynne, "Variational and non-variational eigenvalues of the $p$-Laplacian," Journal of Differential Equations, vol. 244, no. 1, pp. 24-39, 2008.

[3] P. Drábek and P. Takáč, "On variational eigenvalues of the $p$-Laplacian which are not of LjusternikSchnirelmann type," Journal of the London Mathematical Society, vol. 81, no. 3, pp. 625-649, 2010.

[4] N. Ghoussoub, Duality and Perturbation Methods in Critical Point Theory, vol. 107 of Cambridge Tracts in Mathematics, Cambridge University Press, Cambridge, UK, 1993.

[5] M. Struwe, Variational Methods, Springer, Berlin, Germany, 2nd edition, 1996.

[6] P. Drábek and S. Robinson, "Resonance problems for the p-Laplacian," Journal of Functional Analysis, vol. 169, no. 1, pp. 189-200, 1999.

[7] A. Szulkin, "Ljusternik-Schnirelmann theory on $C^{1}$-manifolds," Annales de l'Institut Henri Poincaré, vol. 5, no. 2, pp. 119-139, 1988. 


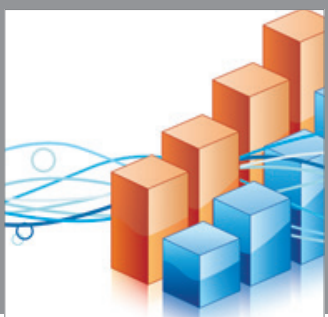

Advances in

Operations Research

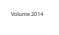

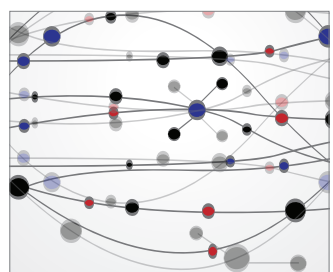

\section{The Scientific} World Journal
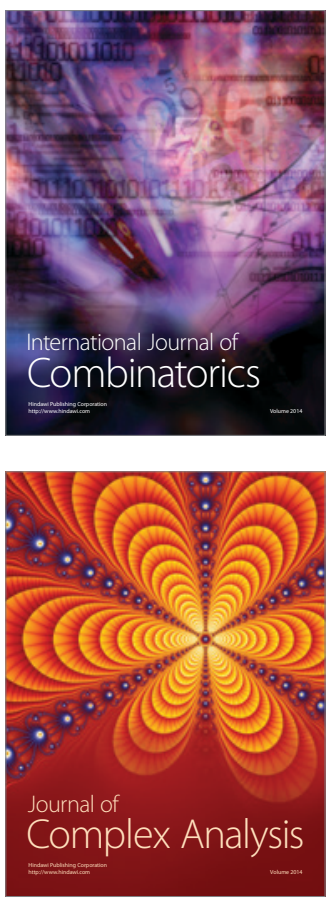

International Journal of

Mathematics and

Mathematical

Sciences
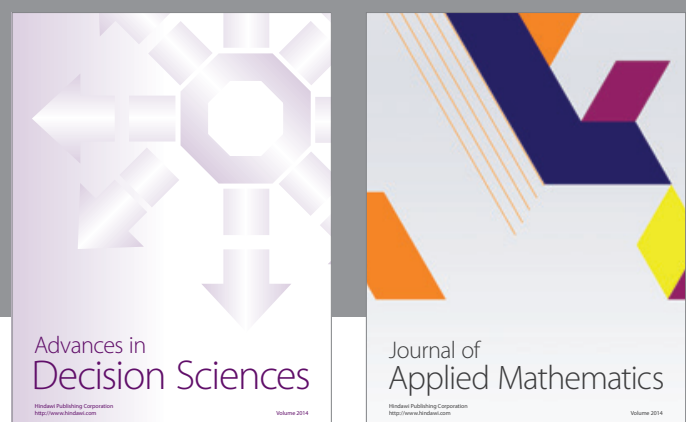

Journal of

Applied Mathematics
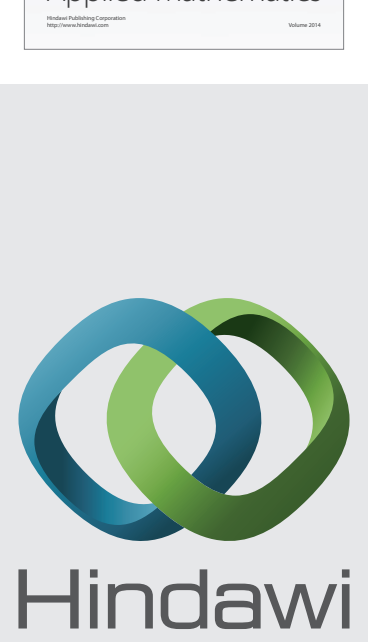

Submit your manuscripts at http://www.hindawi.com
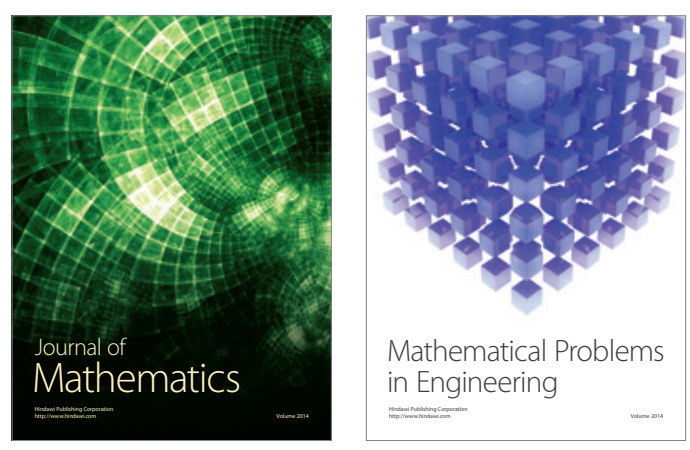

Mathematical Problems in Engineering
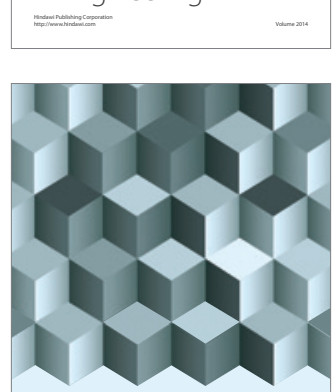

Journal of

Function Spaces
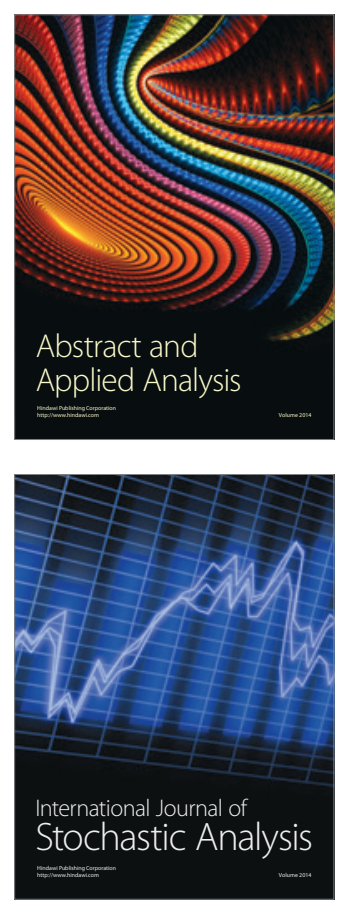

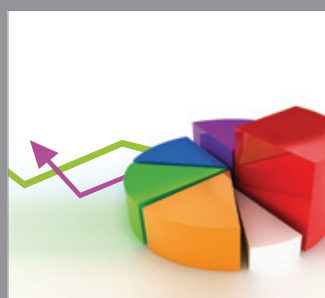

ournal of

Probability and Statistics

Promensencen
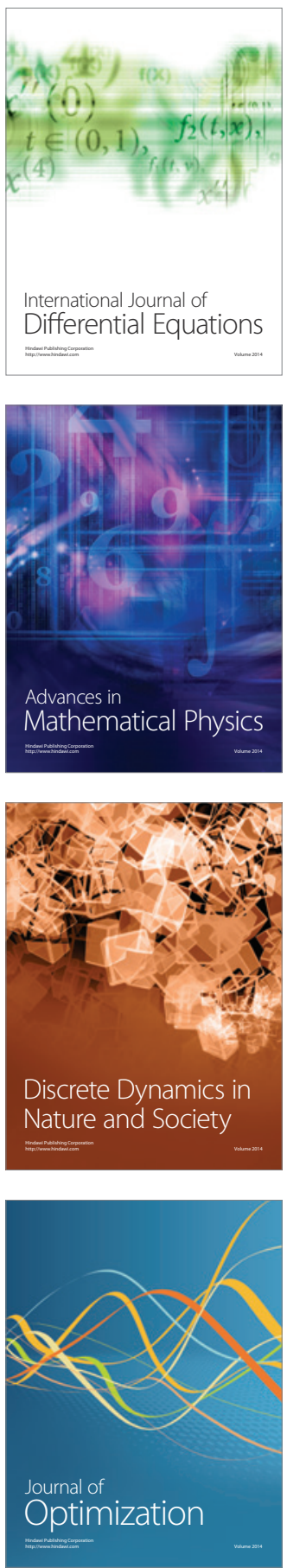